\title{
LAS REALIZACIONES DE LO «ARCAICO» EN EL MUNDO IBÉRICO
}

\author{
POR \\ P. CABRERA, F. CROISSANT, T. CHAPA, R. OLMOS, A. PEREA, L. PRADOS, \\ P. ROUILLARD, A. ROUVERET, A. SCHNAPP Y T. TORTOSA
}

\begin{abstract}
RESUMEN
Se presenta el informe preliminar elaborado por un equipo hispanofrancés sobre las implicaciones de lo llamado "arcaico" en la cultura ibérica: su concepto, su historiografia y su relación con otras columnas mediterráneas. Se tratan aspectos particulares: bronces griegos e ibéricos, joyeria orientalizante, escultura local, cerámica, problemas iconográficos, etc. Se analizan también diferentes formas de comercio en época temprana con las áreas mediterráneas. El trabajo en equipo se desarrollará en el futuro.
\end{abstract}

\section{SUMMARY}

This joint paper presents the preliminar report of a French-Spanish team working on the implications of the so called "archaism" in the Iberian Culture: concepts. historiagraphy and relationship with other Mediterranean areas. Outstanding topics are considered: Greek and Iberian bronzes, Orientalizing jewellery, local sculpture, pottery, iconography, etc. Different exchange and trade techniques between the Peninsula and the East Mediterranean during the Orientalizing period is also analysed. Additional publications will be further presenied.

El equipo hispanofrancés que se ocupa actualmente de una revisión del concepto de lo arcaico en su relación con el mundo ibérico, ha desarrollado un seminario de trabajo en la Casa de Velázquez de Madrid el jueves 3 de diciembre de 1992. Han participado en este seminario Paloma Cabrera (M.A.N., Madrid), Francis Croissant (Univ. Paris, I), Teresa Chapa (Univ. Complutense), Alicia Perea (M.A.N., Madrid), Lurdes Prados (U.A.M., Madrid), Alain Schnapp (Univ. París, I), Agnès Rouveret (Univ. París-Nanterre) y Trinidad Tortosa (C.S.I.C., Madrid). Actuaron como moderadores Pierre Rouillard (C.N.R.S.) y Ricardo Olmos (C.S.I.C.). El trabajo se integra en el proyecto de investigación conjunto «Griegos e Iberos en época arcaica», aprobado por el C.N.R.S.-C.S.I.C. para 1992 y renovado en 1993.

El principal objetivo de este proyecto se centra en la revisión del concepto de arcaismo tradicionalmente aplicado al arte ibérico. Nos planteamos previamente si tiene o no sentido hablar de un arte iberogriego y en qué términos cabe utilizar el uso o usos de este concepto. Como paso previo a la investigación en este primer seminario, nos hemos centrado en una presentación y revisión de materiales correspondientes grosso modo a una supuesta fase 
arcaica hallados en la peninsula ibérica, partiendo de manera puramente convencional de los paradigmas cronológicos de Grecia. En esta revisión, hemos discutido diversos grupos temáticos bajo sus diferentes categorias clasificatorias.

Paloma Cabrera ha presentado en un análisis actualizado la complejidad de la presencia griega en el extremo occidente. Su revisión es a un tiempo conceptual e historiográfica. Se exige un uso más matizado de los términos (presencia y no colonización, diversidad de los lugares comerciales y de los modos de intercambio, redefinición de las categorias de la praxis comercial arcaica incluyendo la misma - tan problemática - del comercio aristocrático, del papel comercial de los santuarios y de las anteriores pautas fenicias). Los nuevos hallazgos replantean en cada yacimiento concreto una nueva visión del comercio arcaico. Al tradicional problema de las tipologías y de las propuestas clasificatorias $-\mathrm{y}$ sus revisiones cronológicas: "copas jonias"- se contrapone hoy el interés creciente por más precisa de los talleres productores. Ello conlleva una nueva reflexión sobre las fuentes (presencia focea), e incluso ir más allá de aquello que las fuentes no transmiten expresamente ( $¿$ es posible hablar de una diversidad comercial jonia, frente a la tácitamente aceptada exclusividad o preponderancia focea?). Deberá preocupar más la aún desconocida vertiente de las producciones "coloniales», así como el modo de distribución de los productos importados en las rutas del interior. Los conjuntos no se estudian ya aislados, por «etnias», sino asociados a otros productos concominantes que llegan con el comercio (debate del bucchero etrusco), etc. En definitiva, Paloma Cabrera despliega en su síntesis toda la complejidad, llena aún de interrogantes, de la presencia griega arcaica.

Lurdes Prados ha introducido el tema de los bronces «arcaicos» más allá de su simplicidad cronológica, en la dialéctica de su dinámica evolutiva. El tema de su vinculación étnica - procedencia fenicia o griega de los estímulos- quedaría superada en el nuevo significado de su realización occidental. Ha considerado diacrónicamente tres grandes estadios. En un primer momento, la presencia de bronces se vincularia a importaciones predominantemente orientales y a santuarios costeros. El debate se establece aún al definir aquellas importaciones de los objetos realizados en la península en su doble vertiente: como productos de un artesanado colonial o como realizaciones propiamente locales, indigenas. Este proceso dialéctico entraña una vertiente productiva - una relación artesanal-e ideológico-religiosa. Esta última es mecánica: implica la significación del bronce en su propio contexto social que le justifica y explica. Ello nos sitúa en un segundo estudio. Frente al santuario costero debe considerarse la existencia de bronces, aún aislados, en el interior (ejemplos del "Guerrero» de Medina de las Torres, o el Jinete de la Bastida de los Alcuses). Nuevos conceptos, incorporando algunas propuestas recientes de $\mathrm{M}$. Almagro Gorbea, entran en juego en el ámbito de la lectura simbólica: ¿cabe hoy ver en algunos de estos bronces «lusitanos» la vieja divinidad oriental (el llamado "Smiting God») o un sincretismo local; expresión de una monarquia divinizada; horoización de un antepasado? Un tercer momento vincula ya los bronces a santuarios ibéricos: implica una generalización. Cada santuario parece definir su propia tipologia especializada: Collado de los Jardines - exvotos masculinos y abundancia de jinetes-; Castellar - predominio femenino y ausencia de jinetes-, etc. L. Prados trata de definir tipos y funciones, con la compleja relación del objeto con el oferante, de la imagen con la realidad. Y trata también de estudiarlos en su contexto histórico geográfico: rutas, emplazamiento y función integradora desde el punto de vista social y territorial de los santuarios, etc. Otros problemas - cronológicos, evolutivos y de producción artesanal- han quedado apuntados en esta exposición.

Teresa Chapa plantea una síntesis de la gran plástica ibérica en piedra, distinguiendo previamente entre el carácter hipotético de una posible etapa arcaica (o, más asépticamente antigua) frente a una perduración de determinados rasgos arcaicos (lo arcaizante). Frente a posiciones "colonialistas" considera la necesidad de buscar en el propio mundo ibérico las razo- 
nes de la existencia de esta plástica, aceptando la foraneidad de sus formas. La adopción en piedra de la gran escultura en la etapa antigua (a partir del siglo vi y a lo largo del v a. C.) debe justificarse internamente en el nuevo sistema socioeconómico de la aristocracia ibérica que busca un modo de expresión a través de la escultura. Hay que asociar los monumentos escultóricos y su proyección mítico-iconográfica a la reestructuración del territorio (cf., por ejemplo, en Jaén, la ordenación del territorio en torno a los ejes de Cástulo y Porcuna). El monumento de Pozo Moro es, ante todo, una referencia social y territorial. Formalmente revela una raigambre oriental - o carencia de uniformidaden los tanteos de los origenes. La eclosión de formas (todo el repertorio animalistico-fabuloso), de influjos diversos (fundamentalmente griegos y orientales) y de posibles caminos debe corresponder, en gran medida, a la paralela eclosión de las aristocracias en el nuevo espacio ibérico. La ausencia de una evolución clara junto a los matices diferenciales de una dialéctica local caracterizan este temprano momento que sólo analógicamente podríamos definir como arcaismo ibérico. En ningún caso sirve aqui el concepto de arcaico tal como se ve desde la lectura griega.

Francis Croissant, en una revisión que se apoya en datos aportados por Pierre Rouillard, nos ofrece la reflexión de un especialista en mundo griego, en plástica helénica. Analiza, pues, el problema desde un punto de observación externo a lo ibérico. Parte del interrogante de qué elementos griegos ha podido ver el artesano ibérico. $\mathrm{Y}$ tras esta pregunta - ihan visto los artesanos iberos obras griegas?-revisa individualmente los supuestos bronces considerados de un modo u otro griegos o grequizantes hallados en España: sátiro del Llano de la Consolidación (laconio); el centauro de Royos, para el que plantea Croissant la presencia de ciertos rasgos específicos peculiares que nos sitúan en el límite de lo griego y de lo indígena; el sileno danzante de Capilla, obra claramente no griega y tal vez ni siquiera arcaica; "guerrero de Cádiz», etc. Todo ello replantea una revisión - basada en análisis estilísticosde los posibles influjos, de los modelos y de los estimulos diversos que confluyen en las realizaciones locales.

Alicia Perea centra su análisis en diversos ejemplos concretos -diacrónicos- de la orfebreria peninsular, considerándola desde las consecuencias que se pueden extraer de un análisis prioritario pero no exclusivamente técnico. Un ejemplo como el tesoro de Villena del Bronce Final deberia, de una vez por todas, hacernos superar el viejo prejuicio historiográfico de la ineptitud del indigena, vigente desde comienzos de siglo. El periodo «orientalizante" o tartésico, ejemplificado en Aliseda, supone una ruptura tecnológica, una innovación. La disyuntiva artesanos fenicios e indigenas se resuelve en las implicaciones de una relación estrecha, requeridos por toda transmisión tecnológica. Técnicas tan definidas como el granulado o la filigrana sólo han podido realizarse por medio de un contacto personal entre artesanos. Este contacto explica la rápida aceptación indigena de nuevas técnicas. Tesoros como el de Serranilla o Segura de León - situables en una fase ibérica antigua o "arcaica"- apuntan a la continuidad tecnológica. No así iconográfica y tipologia con innovaciones. Finalmente, la orfebreria de Cancho Roano nos sitúa en un importante centro de actividad politica, religiosa y económica que permite pensar en la existencia de un taller de orfebrería con amplia proyección expansiva y modélica hacia el ulterior mundo ibérico.

Trinidad Tortosa propone unas reflexiones sobre el nacimiento de la imagen cerámica en el mundo ibérico. ¿Parten determinados estímulos decorativos ibéricos de una anterior estimulo orientalizante y «colonial»? Desde esta perspectiva, $y$ en determinadas zonas - sureste andaluz, mundo levantino--, analiza determinados elementos, tanto bajo una posibilidad evolutiva, como los residuos de una anterior herencia fenicia. Se plantean, más que respuestas, cuestiones puntuales considerándose el tránsito hacia determinadas cerámicas ibéricas a partir de los rastros de elementos orientalizantes (por ejemplo, casos de Peña Negra y Saladares, en Alicante). En esta línea de una perdida tradición orientalizante queda planteada conjeturalmente la existencia de elementos ico- 
nográficos antiguos en la cerámica temprana iberica, como es el peculiar caso de las representaciones animales, metópadas, en algunos ejemplos de Elche.

El seminario sobre arcaismo se complementa con un "excursus" historiográfico de Alain Schnapp sobre la figura de Pedro Bosch Gimpera. Ofrece Schanpp unos elementos de reflexión sobre la peculiar proyección universal de la figura cientifica y politica de Bosch, que a través de una visión cientifica particularizada en la arqueología catalana y, seguidamente, de la península ibérica trasciende y logra el acceso a una arqueologia global y europea. Esta amplia visión europea se gesta principalmente durante sus años de actividad en Barcelona, y se explica en la perspectiva que la Cataluña de la época ofrece como microcosmos de la cultura europea. Analiza el papel unificador en la investigación de Bosch desde su puesto politico (visión de las excavaciones, de los Museos y de la Universidad), su vertiente teórica (como comunicador, formador de una escuela, como divulgador de unas ideas científicas) para concluir, sobre todo, con el impulso globalizador que multiplica su faceta universitaria e internacionalista tras el exilio en Méjico: incorporará allí la visión antropológica a la arqueológica enlazándola con una más rica visión del patrimonio.

Sendas conferencias de Ricardo Olmos y Agnès Rouveret, dirigidas a un público más amplio, cierran la sesión científica y tratan de ofrecer respectivamente dos modelos de aproximación diferentes de interpretación iconográfica: por un lado, la lectura interna de la imagen ibérica, generalmente descontextualizada $y$ sin el apoyo de referencias textuales ( $R$. Olmos); por otro, el análisis de las tumbas lucanas a través de su articulación sintáctica en un sistema de signos que nos ofrece una relación entre los diferentes talleres lucanos que decoran sus paredes.

En el trabajo de este primer año, el equipo de investigadores francoespañoles que redactamos este informe, hemos tratado de ofrecer los datos esenciales de un debate que habrá que desarrollar en el futuro para definir el problema básico que centra hoy nuestra investigación: por un lado, definir el grado de participación respecto a otras culturas mediterráneas del fenómeno arcaico; por otro, establecer los parámetros diferenciales, es decir, la peculiaridad de lo que de un modo genérico y convencional corresponderia con un supuesto período arcaico de las culturas ibéricas. ¿Es lícito aplicar en nuestro caso un concepto de un fenómeno prestado y modelado historiográficamente sobre modelos griegos? ¿Cuáles son los rasgos de ese proceso mediterráneo en el extremo occidente? Una discusión plural en el futuro deberá profundizar más en los diversos aspectos hoy aquí planteados.

Diciembre de 1992 